Commun. math. Phys. 29, 105-111 (1973)

(C) by Springer-Verlag 1973

\title{
Two Examples Illustrating the Differences between Classical and Quantum Mechanics
}

\author{
Jeffrey Rauch* \\ Department of Mathematics, The University of Michigan, Ann Arbor, Michigan \\ Michael Reed \\ Department of Mathematics, Princeton University, Princeton, New Jersey
}

Received August 1, 1972

\begin{abstract}
Two examples are presented: The first shows that a potential $V(x)$ can be in the limit circle case at $\infty$ even if the classical travel time to $\infty$ is infinite. The second shows that $V(x)$ can be in the limit point case at $\infty$ even though the classical travel time to infinity is finite. The first example illustrates the reflection of quantum waves at sharp steps. The second example illustrates the tunnel effect.
\end{abstract}

In this paper we give two examples of motion on a half-line which illustrate two physical differences between classical and quantum mechanics. It is useful to study the half-line case since the necessary techniques and estimates are elementary and the complications which arise in higher dimensions are absent. We say that a potential $V(x)$ is classically complete at $\infty$ if the classical travel time to infinity is infinite for all initial conditions. We say that $V(x)$ is quantum mechanically complete at $\infty$ if the differential operator $-\frac{1}{2 m} \frac{d^{2}}{d x^{2}}+V(x)$ is in the limit point case at $\infty$. At first glance it seems that the two notions of completeness might be the same since $-\frac{1}{2 m} \frac{d^{2}}{d x^{2}}+V(x)$ is in the limit point case at $\infty$ if one need not specify boundary conditions at $\infty$. In a rough intuitive sense, this should happen if the classical travel time is infinite. But, in fact, this rough intuition is correct only if the derivatives of $V(x)$ are "small" compared to $V(x)$. We present two examples illustrating this fact. In the first example, $V(x)$ is classically incomplete at $\infty$ but quantum mechanically complete; in the second, $V(x)$ is classically complete at $\infty$ but quantum mechanically incomplete. The examples

\footnotetext{
* This research was partially supported by the National Science Foundation under Grant GP-34260.

For help with the diagrams, the authors thank Bob Johnson.

$\&$ Commun. math. Phys., Vol. 29
} 
were suggested by Nelson in unpublished lectures at Princeton. The two examples are interesting because in both cases the differences arise from quantum phenomena not found in classical mechanics; namely, the reflection of quantum waves at sharp steps in Example 1 and the tunnel effect in Example 2. This illustrates the point that questions which at first appear to be merely technical mathematical problems often turn out to be closely related to the physics of the situation being described.

In the following brief discussion we always assume that $V(x)$ is a real-valued continuously differentiable function on $(0, \infty)$. If $x(t)$ and $v(t)$ are position and velocity of a classical particle moving in the potential $V$, the Hamiltonian is $H(x, v)=\frac{1}{2} m v^{2}+V(x)$ and the classical equations of motion are:

$$
\dot{x}(t)=v(t), \quad \dot{v}(t)=-\frac{1}{m} V^{\prime}(x(t)) .
$$

For each pair $\left\langle x_{0}, v_{0}\right\rangle$, standard arguments give the existence and uniqueness of a solution $\langle x(t), v(t)\rangle$ for $\left|t-t_{0}\right|$ sufficiently small and satisfying $x\left(t_{0}\right)=x_{0}, v\left(t_{0}\right)=v_{0}$. An elementary argument using uniqueness and the conservation of energy shows that if a global solution (i.e., for all $t>t_{0}$ ) fails to exist, then there is a $\tau>t_{0}$ so that either $\operatorname{Lim}_{t \rightarrow \tau} x(t)=0$ or $\operatorname{Lim}_{t \rightarrow \tau} x(t)=\infty$. The second alternative can only occur if $\sup _{x \geqq 1} V(x)<\infty$ and if the classical travel time (from $x=1$ ), $\int_{1}^{\infty} \frac{d x}{\sqrt{E-V(x)}}$, is finite for $E>\sup _{x \geqq 1} V(x)$. If the second alternative holds we say that $V(x)$ is classically incomplete at $\infty$.

In the quantum mechanical case, let $H$ be the linear operator $-\frac{1}{2 m} \frac{d^{2}}{d x^{2}}+V$ defined on the dense set $C_{0}^{\infty}(0, \infty)$ in $L^{2}(0, \infty) . H$ is essentially self-adjoint on $C_{0}^{\infty}(0, \infty)$ if and only if the ordinary differential equation

$$
-\frac{1}{2 m} \phi^{\prime \prime}(x)+V(x) \phi(x)=0
$$

is the limit point case both at 0 and at $\infty$; i.e., if and only if exactly one nontrivial solution (up to scalar multiples) of (ii) is square integrable near 0 and exactly one is square integrable near $\infty$. (For proofs, see $[1$, Chapter 9] or [2, $\$$ XIII. 2].) If $H$ is not essentially self-adjoint, one must choose a self-adjoint extension by fixing boundary conditions at 0 or at $\infty$ (or both). In the case where exactly one solution of (ii) is square integrable near $\infty$ (limit point case) we say that $V(x)$ is quantum mechanically complete at $\infty$. 
As mentioned above, a rough intuition says that $V(x)$ should be quantum mechanically complete at $\infty$ if and only if the classical travel time to infinity is infinite because then one should not have to specify boundary conditions at $\infty$. The following result shows that this is in fact true if the derivatives of $V$ are small compared to $V$.

Theorem (Wintner [4]). Let $V(x)$ be a twice continuously differentiable function on $(0, \infty)$ which satisfies $V(x) \rightarrow-\infty$ as $x \rightarrow \infty$, and suppose that

$$
\int_{c}^{\infty}\left|\left(\frac{\left[(-V)^{1 / 2}\right]^{\prime}}{(-V)^{3 / 4}}\right)^{\prime}(-V)^{-1 / 4}\right| d x<\infty
$$

for some $c>0$. Then $V(x)$ is quantum mechanically complete at $\infty$ if and only if $V(x)$ is classically complete at $\infty$.

We now present two examples which show that if $V^{\prime}(x)$ is not small compared to $V(x)$, then the classical and quantum notions of completeness at $\infty$ are independent.

Example 1 ( $V$ is classically incomplete but quantum mechanically complete at $\infty$ ). The potential $V(x)$ will be a sequence of plateaus at heights $-\pi^{2} k^{4}$ smoothly connected by steep cliffs on the short intervals $\left(\alpha_{k}, \beta_{k}\right)$ about the points $x=k$; see Fig. 1. Since $\int_{0}^{\infty} \frac{d x}{\sqrt{-V(x)}}<\infty$, the classical motion under this potential is incomplete at $\infty$. However, if the steps are sharp enough the quantum motion will be complete. The reason for this behavior is that part of the quantum wave is reflected at each of the sharp steps and the steps are arranged so that most of the wave never escapes to infinity.

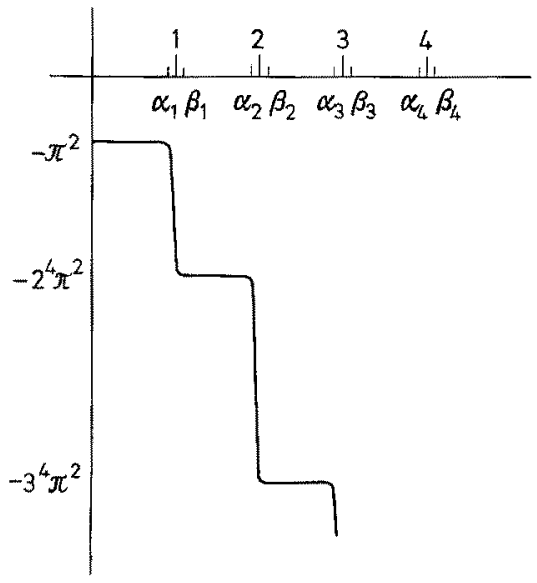

Fig. 1 
What we need to do is to construct $V(x)$ so that there is a solution of (ii) which is not in $L^{2}$ near infinity. To understand the idea, consider the case of infinitely sharp steps, i.e. $\alpha_{k}=k=\beta_{k}$. For $x \in(n-1, n)$ let $\varphi(x)=\cos \left(n^{2} \pi c x-\pi\right)$ with $c=\sqrt{2 m}$. Then $\varphi$ satisfies (ii) except at the integers and clearly $\phi \notin L^{2}(0, \infty)$. In the following construction we just smooth out the sharp steps on the intervals $\left(\alpha_{k}, \beta_{k}\right)$ in such a way that the corresponding solution $\phi$ remains outside $L^{2}$.

On each short interval $\left(\alpha_{k}, \beta_{k}\right), k=1,2,3, \ldots$, we define $V(x)$ to be any monotone decreasing function so that $V(x)$ is continuously differentiable on $(0, \infty)$. We now show how to choose the $\alpha_{k}$ and $\beta_{k}$. Take $\alpha_{1}=1$ and define $\phi(x)=\cos \left(\pi_{c} x-\pi\right)$ on $(0,1]$. At $x=1, \phi(1)=1$ and $\phi^{\prime}(1)=0$. We want to choose $\beta_{1}$ so that the solution has not descended much at $\beta_{1}$. Since $V(x)<0$ the solution $\phi(x)$ of (ii) which equals $\cos \left(\pi_{c} x-\pi\right)$ on $(0,1]$ will be concave downward between 1 and its next zero, $r_{1}$. On the interval $I=\left(1, \min \left\{r_{1}, 2\right\}\right), \phi(x)$ satisfies

$$
\phi(x)-1=2 m \int_{1}^{x}\left(\int_{1}^{s} V(t) \phi(t) d t\right) d s
$$

which implies that

$$
\begin{aligned}
|\phi(x)-1| & \leqq \frac{(x-1)^{2}}{2}\left(\sup _{I}|V(t)|\right)\left(\sup _{I}|\phi(t)|\right)(2 m) \\
& \leqq 2^{4} \pi^{2} \frac{(x-1)^{2}}{2}(2 m) .
\end{aligned}
$$

Choose $\beta_{1}$ so close to 1 that $\left|\phi\left(\beta_{1}\right)-1\right| \leqq 1 / 4$, the point being that this estimate holds no matter how we patch together the steps just as long as $V(x)$ is greater than $-2^{4} \pi^{2}$ in $\left(\alpha_{1}, \beta_{1}\right)$. On $\left(\beta_{1}, \alpha_{2}\right)$ the solution has the form $\phi(x)=A_{2} \cos \left(2^{2} \pi_{c} x-\gamma\right)$ where we must have $\left|A_{2}\right| \geqq 1-\frac{1}{4}$ since $\phi\left(\beta_{1}\right) \geqq 1-\frac{1}{4}$. Now, choose $\alpha_{2}$ to be the closest point to 2 where $A_{2} \cos \left(2^{2} \pi_{c} x-\gamma\right)$ has a maximum. At $\alpha_{2}, \phi\left(\alpha_{2}\right) \geqq 1-\frac{1}{4}$, so using the same idea as above we can choose $\beta_{2}$ so that $\phi\left(\beta_{2}\right) \geqq 1-\frac{1}{4}-\frac{1}{8}$. Continuing in this manner we construct a solution $\phi(x)$ of (ii) so that $\phi(x)$ $=A_{n} \cos \left(n^{2} \pi_{c} x-\gamma_{n}\right)$ on $\left(\beta_{n-1}, \alpha_{n}\right)$ and such that $\left|\alpha_{n}-n\right|+\left|\beta_{n}-n\right| \rightarrow 0$ and $\left|A_{n}\right| \geqq 1 / 2$ for all $n$. Thus, $\phi(x)$ will not be in $L^{2}(0, \infty)$, which implies that $V(x)$ is in the limit point case at $\infty$, i.e. $V(x)$ is quantum mechanically complete at $\infty$.

Example $2(V(x)$ is classically complete but quantum mechanically incomplete at $\infty)$. The potential $V(x)$ will be of the form $\frac{1}{x^{2}}-x^{4}+\sum_{k=1}^{\infty} \sigma_{k}(x)$ where $\sigma_{k}(x)$ is a very narrow smooth spike centered at $x=k$ with radius of support $d_{k}$. The spikes are chosen so that $V(k)=k$; see Fig. 2. Since $V(x)$ is not bounded from above near infinity the classical motion is 


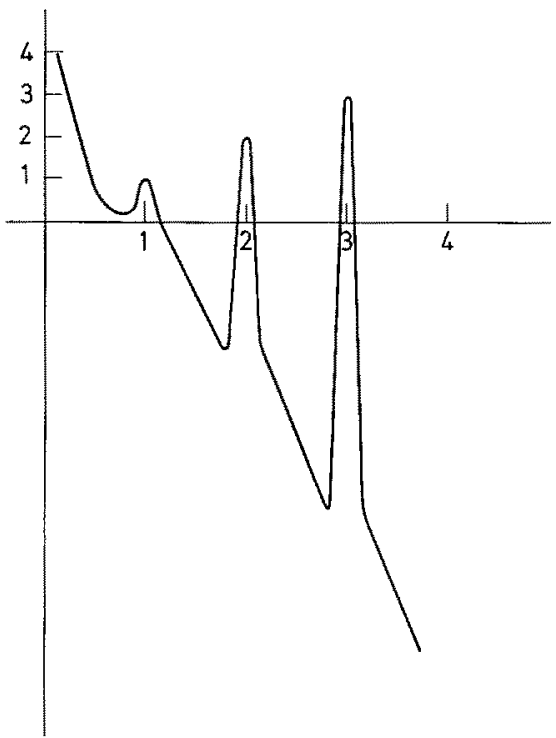

Fig. 2

complete at infinity. We will show that if the supports of the spikes are chosen to be narrow enough, then $-\frac{1}{2 m} \frac{d^{2}}{d x^{2}}+\frac{1}{x^{2}}-x^{4}+\sum_{k=1}^{\infty} \sigma_{k}(x)$ will not be essentially self-adjoint on $C_{0}^{\infty}(0, \infty)$. Since $V(x)$ is in the limit point case at $x=0[2$, p. 1415], this shows that $V(x)$ can not be in the limit point case $\infty$, i.e., $V(x)$ is not quantum mechanically complete at $\infty$. The physical reason for this behavior is that if the spikes are narrow enough, the quantum particle can tunnel through even though the classical particle is turned back. Thus to a quantum mechanical particle the potential $V$ is not very different from $V_{1}=\frac{1}{x^{2}}-x^{4}$ which, by Wintner's theorem, is not quantum mechanically complete at $\infty$.

We will show that if the supports of the spikes are small enough there is an $a, 0 \leqq a<1$, so that

$$
\left\|\sum_{k=1}^{\infty} \sigma_{k} \phi\right\|^{2} \leqq a^{2}\left\|-\frac{1}{2 m} \phi^{\prime \prime}+V_{1} \phi\right\|^{2}+b^{2}\|\phi\|^{2}
$$

for all $\phi \in C_{0}^{\infty}(0, \infty)$. The symmetric form of the Kato-Rellich theorem [3, Chapter V, Theorem 4.5] then implies that $-\frac{1}{2 m} \frac{d^{2}}{d x^{2}}+V_{1}$ and $-\frac{1}{2 m} \frac{d^{2}}{d x^{2}}+V_{1}+\sum_{k=1}^{\infty} \sigma_{k}$ are either both essentially self-adjoint on 
$C_{0}^{\infty}(0, \infty)$ or both not essentially self-adjoint. Since $-\frac{1}{2 m} \frac{d^{2}}{d x^{2}}+V_{1}$ is not essentially self-adjoint, it follows that $-\frac{1}{2 m} \frac{d^{2}}{d x^{2}}+V_{1}+\sum_{k=1}^{\infty} \sigma_{k}$ is not essentially self-adjoint.

To prove (iii) we use a simple a priori estimate. For each positive integer $m$ and each $x_{0} \in \mathbb{R}$, let $I_{m}^{x_{0}}=\left\{x|| x-x_{0} \mid \leqq 1 / m\right\}$. Then, there is a constant $C$ (independent of $x_{0}$ ) such that

$$
\sup _{x \in I_{4}^{x_{0}}}|\phi|^{2} \leqq C\left(\left\|\phi^{\prime \prime}\right\|_{L^{2}\left(\left(_{2}^{x_{0}}\right)\right.}^{2}+\|\phi\|_{L^{2}\left(I_{2}^{x_{0}}\right)}^{2}\right)
$$

for all $\phi \in C_{0}^{\infty}(\mathbb{R})$. To prove (iv), let $\eta$ be a $C^{\infty}$ function with support in the interval $\left(-\frac{1}{2}, \frac{1}{2}\right)$ which is indentically one on $\left(-\frac{1}{4}, \frac{1}{4}\right)$. We have

$$
\phi(x)=\int_{x_{0}-\frac{1}{2}}^{x} \int_{x_{0}-\frac{1}{2}}^{t}\left(\eta\left(s-x_{0}\right) \phi(s)\right)^{\prime \prime} d s d t
$$

and (iv) follows by carrying out the differentiations, integrating by parts in the $\eta^{\prime} \phi^{\prime}$ term, and then using the Schwarz inequality.

Let $\sigma_{k}(x)$ be a non-negative $C^{\infty}$ function with support in the interval $\left(k-d_{k}, k+d_{k}\right)$ with $d_{k} \leqq 1 / 4$. Furthermore let $\sigma_{k}$ reach a maximum value of $-\frac{1}{k^{2}}+k^{4}+k=M_{k}$ at $x=k$. Then for all $\phi \in C_{0}^{\infty}(0, \infty)$

$$
\begin{aligned}
&\left\|\sigma_{k} \phi\right\|^{2} \leqq M_{k}^{2} d_{k}\left(\sup _{x \in I_{4}^{k}}|\phi|^{2}\right) \leqq 2 M_{k}^{2} d_{k} C\left(\left\|\phi^{\prime \prime}\right\|_{L^{2}\left(l_{2}^{k}\right)}^{2}+\|\phi\|_{L^{2}\left(I_{2}^{k}\right)}^{2}\right) \\
& \leqq 2 M_{k}^{2} d_{k} C\left[(2 m)^{2}\left\|-\frac{1}{2 m} \phi^{\prime \prime}+V_{1} \phi\right\|_{L^{2}\left(I_{2}^{k}\right)}^{2}+(2 m)^{2}\left\|V_{1} \phi\right\|_{L^{2}\left(I_{2}^{k}\right)}^{2}\right. \\
&\left.+\|\phi\|_{L^{2}\left(I_{2}^{k}\right)}^{2}\right] .
\end{aligned}
$$

Now choose $d_{k}$ so that $2 M_{k}^{2} d_{k} C(2 m)^{2} \leqq \frac{1}{2}$ and so that

Then

$$
2 M_{k}^{2} d_{k} C(2 m)^{2} \sup _{x \in I_{2}^{k}}\left|V_{1}\right|^{2} \leqq 1 .
$$

$$
\begin{aligned}
\left\|\sum_{k=1}^{\infty} \sigma_{k} \phi\right\|^{2} & =\sum_{k=1}^{\infty}\left\|\sigma_{k} \phi\right\|^{2} \\
& \leqq \sum_{k=1}^{\infty} \frac{1}{2}\left\|-\frac{1}{2 m} \phi^{\prime \prime}+V_{1} \phi\right\|_{L^{2}\left(I_{2}^{k}\right)}^{2}+2\|\phi\|_{L^{2}\left(I_{2}^{k}\right)}^{2} \\
& =\frac{1}{2}\left\|-\frac{1}{2 m} \phi^{\prime \prime}+V_{1} \phi\right\|^{2}+2\|\phi\|^{2} .
\end{aligned}
$$


This proves the estimate (iii) which implies as discussed above that $V(x)$ is not in the limit point case at $\infty$.

As an interesting exercise, the reader is invited to work Example 1 using the technique of Example 2 and Example 2 with the technique of Example 1.

\section{References}

1. Coddington, E., Levinson, $\mathrm{N}$.: Theory of ordinary differential equations. New York: McGraw-Hill, 1955.

2. Dunford, N., Schwartz,J.T.: Linear operators, II. New York: Interscience 1963.

3. Kato, T.: Perturbation theory for linear operators. Berlin-Heidelberg-New York: Springer 1966.

4. Wintner, A.: On the normalization of characteristic differentials in continuous spectra. Phys. Rev. 72, 516-517 (1947).

Jeffrey Rauch

Department of Mathematics

The University of Michigan

Ann Arbor, Mich. 48104

USA
Michael Reed

Department of Mathematics

Princeton University

Princeton, N. J. 08540

USA 